Moral distress as experienced by hospital social workers in South Korea and Australia

\title{
Authors:
}

Patricia Fronek, Lynne Briggs, Myung Hun Kim, Hye Bin Han, Val Quinn, Sungmin Kim and Donna McAuliffe.

\begin{abstract}
This exploratory, qualitative research explored the ethical problems faced by hospital social workers in South Korea and Australia, and what and who influenced their decision making using a focus group design. Although dilemmas of boundaries, confidentiality, self-determination and other complex scenarios found in practice were identified, moral distress, a consequence of the unresolvable conflicts, dominated participants’ narratives. This was particularly the case for the Korean social workers in this sample. A thematic analysis of the data yielded three main themes: 'Under pressure - “It's very uncomfortable”, 'Failing our patients'; and 'Coping and codes'.
\end{abstract}

Key words: Moral distress, ethics, values, hospital social work 
Social work's genesis and ongoing development are grounded in moral concerns (Bisman, 2004). Using a normative philosophical conceptualisation, social work is arguably moral work because members of the profession are committed to a certain moral schema reflected in the shared and socially agreed to values of social justice and respect for people regardless of differences in individual moral beliefs (Cooper, 1966; Gray 2010). While recognising that there are variations between social work codes of ethics, cultures and contexts, shared values are reflected in these codes where they have been developed (Banks, 2012; Congress \& McAuliffe, 2006; Fine \& Teram, 2009).

Although social work may share a common morality, some authors suggest the purpose of social work may have become lost in the bureaucratic nature of codes of ethics (Banks, 2003, 1998; Fine \& Teram, 2009; Weinberg, 2010; Weinberg and Campbell, 2014). In order to achieve credibility and acceptability, it is suggested that codes of ethics have become disciplinary, technocratic and procedural, and in that process the norms that describe and govern behaviour have become disconnected from social work’s purpose (Banks, 2003, 1998; Fine \& Teram, 2009; Weinberg, 2010; Weinberg and Campbell, 2014). The relevance of codes of ethics that essentially provide descriptive understandings of morality and a focus on individual behaviour are questioned. These critiques suggest a greater emphasis on conscience or personal morality and professional identity is needed.

The impetus for the exploratory study reported in this paper arose from this very question about the relevance of codes of ethics to everyday health practice. An opportunity to explore the kinds of challenging ethical situations experienced by 
hospital social workers in two countries that shared similar codes of ethics arose in a conversation about ethics during a visit of the Korean Association of Social Work (KASW) to Australia. Although there is substantial cross disciplinary research that compares health systems, health spending and health outcomes between countries, there is little research that compares the experiences of social workers and the impact of these experiences on social workers and their practices (Brown, 2003; Cacace et al., 2013; Carinci et al., 2015). Understanding commonalities and differences in social work practice is important in the contemporary context where the delivery of health care is increasingly globalised and social work is influenced by international trends (Pockett \& Beddoe, 2015).

As ethics in practice or applied ethics tends to focus on conduct between practitioners and their patients, ethical dilemmas and ethical decision making, the authors expected a predominance of findings related to these aspects of ethics to emerge (Fronek \& Kendall, 2016; McAuliffe, 2014; Reamer, 2005). Although participants reported ethical dilemmas concerned with boundaries, confidentiality, self-determination, and ethical decision making, a surprising finding was the presence of moral distress within themes, the consequence of intractable situations shaped by unjust social policies, poverty and the unethical behaviour of others.

\section{Moral distress}

Most of the literature about moral distress is found in the nursing and psychology literature and is a latecomer to social work research (Weinberg, 2009). According to Weinberg (2009), moral distress is a conceptually flawed concept. In an attempt to unravel the theoretical confusion that beleaguers moral distress, Lützén and Kvist (2012) suggested five distinct concepts. They conceptualised 'moral distress' as a psychological response to moral conflicts that acts as a catalyst for action, 'moral 
stress' as a physiological response, and 'stress of consciousness' as a theological or philosophical response. 'Moral sensitivity' determines behaviour and includes the concepts of moral judgement, moral motivation and moral character. These four concepts describe individual characteristics and responses that determine actions whereas the final concept 'ethical climate' enables or inhibits individual agency. This suggests institutional contexts are an important influence in determining normative moral frameworks within institutions as well as individual actions. Classic moral distress is typically generated by conflicts between personal values and institutional or systemic values (Sunderland, Catalano, Kendall, McAuliffe \& Chenoweth, 2010). The existing literature on moral distress does not explore the impact on practitioners when organisational cultures condone or ignore unethical practices.

Although conceptualisations of moral distress vary depending on the disciplinary focus, there is general agreement that moral distress occurs when a practitioner cannot act according to their moral conscience and suffers negative effects as a result (Austin et al, 2005). Differences lie in how the source of moral conflict is defined and how much moral responsibility for actions and reactions is placed on the individual. Psychological and biological approaches prevalent in healthcare research tend to situate moral distress as a state of not coping and as internal to the individual (Brazil, Kassalainen, Ploeg \& Marshall, 2009; Lützén \& Kvist, 2012; Mänttäri-van der Kuip, 2016; Mukherjee, Brashler, Savage \& Kirschner, 2009). A choice between treatment options where a practitioner is forced to make a difficult decision has been associated with moral distress in nursing (Brazil et al., 2010; Pauly, Varcoe and Storch, 2012). Nathaniel (2002) suggests that although distress is generated by an inability to act according to one’s moral conscience, it is a person's perceived complicity in wrongdoing by not acting that exacerbates distress. 
Conceptualisations that recognize how institutions can limit an individual's ability to act have made way for perspectives that suggest moral distress is caused by an inability on the part of the individual to satisfy all value positions that might include one's own values, those of the profession, the organisation and the patient (Jameton, 1984; Kälvemark et al., 2004; Pauly, Varcoe and Storch, 2012). This assumes that all value positions are worthy of equal consideration and should be met by the individual. Equally satisfying all positions might be a rare event in social work practice and risks perpetual distress when one considers neoliberal health contexts where organisational goals and cost benefits might outweigh optimum health outcomes for patients. Preferencing dollars over human well-being might not necessarily be perceived as a worthy value position. Pauly et al. (2012) argue that the lack of conceptual and theoretical clarity in moral distress research has perpetuated the emphasis on individual responsibility and endowed a victim status on those professionals who experience it.

Moral distress literature has focused on the negative consequences for individuals such as job dissatisfaction, increased sick leave, demoralisation, helplessness and hopelessness, burnout and generally impaired well-being, and has explored how practitioners manage their impact (Abramovitz 2005; Austin et al., 2005; Mänttärivan der Kuip, 2016; Pauly, Varcoe and Storch, 2012). Practitioners cope through covert or overt resistance, silence, sustaining themselves through client work and collegial support (Fine \& Teram, 2013; Greenslade, McAuliffe \& Chenoweth, 2015). Survival strategies have also tended to have an individual focus with scant research attention to how shared professional norms and values might serve to fortify social work’s moral position and how codes might serve as a practical and cohesionpromoting tool by emphasising shared identity and values. 
Unlike the nursing literature, the source of moral conflict in social work is often located in the tensions between professional ethics and organisational demands rather than choices between practice interventions (Fine \& Teram, 2013; Gallina, 2010; Lynch \& Forde, 2016; Meeuwisse, Scaramuzzino \& Swärd, 2009; Sunderland et al, 2010; Weinberg, 2009). Weinberg (2009) goes further pointing out how theoretical understandings of moral distress have neglected the political and social structures that influence how organisations deliver services and the conflicts that consequently arise for social workers who cannot serve their patients as well as they feel they should. A new critical understanding of moral distress and how it plays out in practice has developed, expanding to incorporate structural and environmental factors and in that process changing how power is understood. Lynch and Forde (2016, p.97) provide a pertinent example of the moral distress caused by the double standards of Australian governments interning asylum seeking children in Nauru on one hand and promoting the protection of Australian children on the other.

\section{Moral distress, ethical dilemmas and ethical decision making}

Albeit through different lenses, distinctions are made between morals, values and ethics in philosophy and social work ethics, and between moral distress and ethical decision making (Bauman, 1993; Gray, 2010; Habermas, 1990; Weinberg, 2009). Drawing on Bauman's (1998) postmodern view of ethics, Gray (2010) asserts that although ethics and values can be shared, morality is ultimately personal which implies that moral distress can only really be determined by the individual it affects. Moral distress is experienced as a consequence of certain dilemmas where ethical decision making processes are insufficient to achieve morally comfortable outcomes (Austin et al., 2005; Weinberg, 2009). Specifically, moral distress refers to the impact of those dilemmas that assault core, shared, professional values that may have serious 
consequences for patients, and where the choices available to social workers cannot mitigate against harm, rather than the cognitive ethical decision making process involved in determining the least harmful action. According to Bauman (1998) the more our actions count the more uncertain we are about moral action. Relying solely on an ethical decision making process to take the least harmful action can function as an attempt to appease one's conscience and to avoid personal responsibility which does little to ease moral concerns (Bauman, 1998; Gray 2010).

Weinberg (2009) argues that in social work, ethical dilemmas play out at the individual level with a narrow focus on ethical decision making, whereas moral distress is deeply connected to the political aspects that influence practice such as the dismantling of the welfare state and a market approach to welfare and social policy. Unlike the concerns of Pauly and colleagues (2012) where individuals are conceptualised as victims, importantly Weinberg (2009) suggests that moral distress experienced by social workers reinforces the value base of the professional group rather than the failure of the individual. Just as codes of ethics could play a direct role in validating and supporting collective social work values, identity and actions that uphold them, it is possible they might also play a practical role in alleviating moral distress by transferring sole responsibility from the individual to the collective. According to Pauly, Varcoe and Storch (2012, p.4), it is clear that "the relationships amongst individual experiences of moral distress, structural determinants of moral distress, moral agency and ethical action are not well understood”.

\section{The research context}

Participants in this study are hospital social workers who practise in two very different health systems in Australia and South Korea. Australia’s health system has roots in both private medicine and the welfare state which has developed into a two- 
tiered health system. Healthcare is funded by state and federal governments - private and public hospital care with some overlap. Privately insured healthcare is expensive, incurs out of pocket expenses, allows the insured choice of physician and hospital with decreased waiting times for some treatments such as surgery, and more treatment options may be available (Duckett \& Willcox, 2011; Seah, Cheong \& Anstey, 2013). State-run public hospitals are currently free to all citizens with the exception of copayments for some pharmaceuticals and medical aids. Non-citizens or those residents with limited visas incur charges even in public facilities. Since the 1980s, health policy has shifted towards managerialism and marketization and the promotion of private health insurance. Although conspicuously absent in the private sector, social workers tend to work in multidisciplinary teams across all institutional and community public health areas providing services to people of all ages and backgrounds with all types of health conditions.

In stark contrast, Koreans paid for all their health care before 1977. Now compulsory coverage exists via employers and a single insurance provider (Jeong, 2011; Kim, 2010). Contributions are high, and benefits are low, leaving many people such as the unemployed uninsured. The national insurance, regulated by the Ministry of Health and Welfare and the National Health Insurance Corporation, is publicly and privately funded (Jeong, 2011). Healthcare is delivered by the private sector, doctors are privately employed and ninety percent of all hospital beds are in private institutions (Jeong, 2011). A medical aid scheme, three to four percent of health coverage, provides more limited services to low-income people. Social workers in South Korea are tasked with assisting those people who are disadvantaged and determining their eligibility for services. There is a heavy reliance on charities to provide basic assistance not available within the health system to uninsured patients. 
Social work associations in both countries have well developed codes of ethics, the Australian Association of Social Workers' (AASW) Code of Ethics (2010) and the Korean Association of Social Workers' (KASW) Code of Ethics (2001). A comparison shows strong similarities in values, principles, responsibilities and standards. There are some differences - notably, the Korean code includes a pledge that commits social workers to its values and principles, to concepts of respect for life and social justice, to put the interests of the public before those of the individual, and to oppose corruption. The pledge implies an action rather than a mere reading of the code and infers gravitas to the commitment made. The Australian code is longer and includes a definition of social work, a guide to decision-making, recognition of the First Peoples of Australia and of cultural safety. The three core values on which the AASW code of ethics is based are respect for persons, social justice, and professional integrity. The AASW code of ethics includes a provision that 'social workers will uphold the ethical values and responsibilities of this code, even though employers' policies or official orders may not be compatible with its provisions' (AASW, 2010, S5.4.1.f). These social work codes are a concrete representation of the value norms of the profession, as well as a prescription for acceptable professional behaviour in institutional contexts.

\section{Methodology}

The Australian authors were responsible for the overall study design, Australian recruitment, Australian data collection and analysis of all Australian and Korean data. The Korean authors were responsible for participant recruitment and data collection in South Korea and for translation. Ethics approval was granted by Griffith University, Queensland Health and the Korean Association of Social Workers (KASW) research ethics committees. 
The study was exploratory and qualitative using a focus group design (Wilkinson, 1998). Given shared values identified in codes of ethics between Korean and Australian social workers and assumed common concerns, there were two aims of this research: 1) to identify the common and different experiences of ethical problems faced by hospital social workers in South Korea and Australia and; 2) to understand the varied and various influences on ethical social work practice and decision making in both countries.

Hospital social workers were recruited from one health district in Australia by the distribution of email invitations by the District's Director of Social Work and by information distributed by the Korean Association of Social Workers in one health district in South Korea. Twenty-three volunteers participated, eleven in South Korea and twelve in Australia. Five Korean social workers were male. All other participants were female. The Korean social workers were generally more experienced practitioners with all but four having practised for over ten years whereas eight Australian participants had less than four years of experience. The remaining Australian participants had eight, sixteen and two with fifteen years of experience. Areas of current practice were similar between groups and included general medicine, mental health, accident and emergency, paediatrics, family, women and children, acute care, orthopaedics, gastroenterology, rehabilitation, mental health, cancer and hospice, organ transplantation and aged care.

One focus group was conducted in South Korea and one in Australia, each approximately two hours in duration. Discussions were recorded and transcribed. Prompt questions were used by two facilitators in each group to facilitate open discussion. At the conclusion of the focus groups, participants were then asked to write down responses to nine questions (for example: What are the three main ethical 
issues you face in practice?; What influenced your decisions?) to provide an opportunity to add additional information. The focus groups were a good size for discussions and the facilitators in each focus group reported that participants were eager to discuss their experiences.

The data collected in both the Australian and the Korean focus groups were analysed together using thematic coding, a method well established for use in such studies (Clark \& Braun, 2006; Denzin, 1994). The codes were then used to develop the common themes that emerged from the groups. Comparisons between country experiences were made within these themes, noting common experiences and differences. Measuring quality in qualitative research is a contentious issue with diverse opinions (O’Reilly \& Parker, 2012). Different indices of quality exist for different approaches and the various frameworks available within the evidence base (Reilly \& Parker, 2012). In an attempt to address this issue, the analysis was initially undertaken by two of the researchers where each individually coded participant response was later re-coded into themes. The researchers in both countries achieved agreement on the themes. Credibility, confirmability and trustworthiness were further addressed by an extended planning period, regular communication between researchers, and documenting a clear audit trail.

\section{Findings}

As noted previously, the findings relating to moral distress were unexpected.

Drawing on practice experience, it was envisaged that social workers would focus on a prevalence of ethical dilemmas in work with patients and decision making. Moral distress concerning certain unresolvable ethical dilemmas was brought to the fore and was associated with ethical cultures, and political and structural influences. The impact of political and structural influences on moral distress was a shared experience 
among participants in both countries but more common and of greater intensity for South Korean social workers.

Moral distress was identified within three dominant themes: the pressure and tensions between organisational and professional goals that both the Korean and Australian social workers experienced during the course of their work (Under pressure - "It's very uncomfortable”); the attribution of a personal sense of failure when unable to provide needed treatment for patients (Failing our patients); and the ways that social workers managed ethical dilemmas, moral conflicts and moral distress (Coping and codes).

\section{Under pressure - "It's very uncomfortable"}

Although Korean and Australian social workers reported different sources of pressure, being under pressure ran beneath the surface for all social workers in this study. Some of their experiences led to moral distress. The Korean social workers' experiences of moral distress were unique to their context and related to the structure of their health system, the corruption that the system makes possible, and the unethical behaviours of others as acted out within that system. Their pledge to reject injustice and corruption and to always work in the interests of clients and the public was taken seriously but this commitment also presented moral challenges. Korean social workers talked about being frequently placed in intractable situations where they were asked to break rules and act against their code of ethics by those in positions of greater authority than themselves. Korean social workers reported using their code of ethics and ethical decision making to support their decisions. However, such value conflicts in day to day practice and little organisational power to exercise real choices led to cumulative moral distress when they were compelled to comply. Sometimes they were able to resist these pressures and other times not. The following 
excerpt typifies dilemmas that led to moral conflicts caused by pressures from within a Korean hospital and external to it.

There are cases where we have internal requests when it comes to service support. Since we are a public institute, we receive calls as often as the city council has civil complaint calls. For example, requests for a free caregiver or asking us to put them [a particular patient who is not eligible] at the top of the waiting list... the administration department says that "we had a call from the city council so please put them at the top of the list". But we try to hold out as long as we can on these situations but sometimes we reluctantly move them forward. This is unfair to other patients.

This excerpt explains the dilemmas that were frequent occurrences for the Korean social workers in this study and led to moral distress. It also offers insight into the environment in which they work where favours and exceptions are expected for those of influence and how their professional values are challenged. The KASW Code of Ethics states they "should not discriminate on the basis of status and should not be subject to unjustified interventions or pressures from inside and outside of organizations”. Yet in the organisational context, the responsibility for fulfilling this obligation rests with individual social workers rather than a focus on systemic accountability or reform in the hospital setting.

Pressure on Korean social workers to act unethically by privileging certain patients also came from practitioners in other disciplines who had vested interests - "A doctor who is a member of the charity fund in the hospital donates considerable amounts of money on a monthly basis and because of this reason, requests financial aid for one of his patients who is ineligible to be considered a candidate for financial support”. One social worker reported direct threats to comply with such requests but did not specify 
the nature of those threats. These scenarios were not simply attributable to the behaviour of individuals rather they were symptomatic of an institutional culture where these practices were normative. Moral distress arose from unsatisfactory outcomes caused by a clash of values between social workers and the organisational cultures in which they are employed as well as the wider health system. Korean social policy, inequities in the system related to user-pay models, a reliance on charities and donations, and how advantage is sought in a flawed system translated to pressure and moral distress felt by social workers at the coal face. Dual roles and conflicts of interest inherent in the system were of particular moral concern. Ethical decision making was unable to resolve moral conflicts that had serious implications for their patients.

In contrast, downward pressure felt by Australian social workers identified in this theme related to workload and managerial policies on lengths of hospitalisations, throughput of patients and discharge processes. Although difficult at times, these scenarios did not necessarily lead to moral distress as actions such as advocacy were usually possible. Assessments or discharge recommendations by Australian social workers were sometimes dismissed when other members of the multidisciplinary team chose to ignore them. For example, one Australian social worker discussing power and 'their place' in the hospital hierarchy said, "You can have social workers saying this person's not suitable to be discharged home, an OT [occupational therapist] thinks the same way, a physio does, but the doctor doesn't, then you know they can still disregard the advice that you've given.” Another said “...but at the end of the day the consultant always has the last say of whether someone stays, or someone goes. That's been my experience.” Their experiences of institutional demands and interdisciplinary conflicts were similar to those reported in research conducted in 
other western countries where such conflicts were reported to cause distress (Austin et al., 2005). In this study, moral distress was experienced when risks to patients existed and advocacy proved ineffective.

When it came to fund raising, the use of telethons to raise money for individual patients was peculiar to Korean hospital social workers. One Korean social worker reported: "When recommending a patient for a telethon, it is difficult to decide whether this recommendation is beneficial since there are differences in the amount of funds collected depending on whether the patients' names are revealed or not during the telethon”. Public exposure perhaps accompanied by a certain amount of shame and unwanted exposure of private difficulties may be the only way a patient and their family can raise money to pay for their healthcare. Korean social workers felt strongly that they were placing their patients in a situation where they may experience adverse consequences and although they may ultimately receive the treatment they need, the risks are not in their patient's best interests. Although decision making processes were exercised in these cases, these processes did not protect the social workers from moral distress.

Another common source of pressure for the Korean social workers was how they were expected to place the interests of the organisation above that of their patients in incidents of medical malpractice or mistakes imposing dilemmas of dual but opposing responsibilities. Korean social workers are expected not to disclose incidents of medical mishaps to patients and their families. Those patients affected (and not told) can file law suits if they suspect medical mishap, something low-income people cannot afford to do. If patients file law suits, they are ineligible for services, therefore to obtain help, people just accept their circumstances and for social workers “....also as an employee of the hospital, we cannot address the problem and a lot of times we 
just turn a blind eye to it" and "So these kind of incidents create a confusion between being a worker who belongs in an organisation but at the same time being a person with personal beliefs and identity. These are the difficulties that I face”.

Moral distress was experienced by Korean social workers as a result of these "pressure" situations, where the system and individual actions in the organisation were in conflict with social work values and resulted in adverse outcomes for patients and could not be satisfactorily resolved through ethical decision making.

\section{Failing our patients}

The impact of a range of social policies filtered down to the practice level as a source of distress. Amongst the most distressing situations described by participants were those perceived as a failure to provide an adequate service to all their patients, universally attributed to lack of resources and the limitations of eligibility, also noted by Lützén and Kvist (2012). Discriminatory attitudes and lack of resources in health and welfare sectors to assist young people at risk, people with mental health conditions, single mothers, the homeless and those people who make poor decisions, affected the capacity of social workers to meet basic patient need leaving a sense of personal failure.

The Korean social workers reported that people in lower socio-economic circumstances could not pay for costly treatment, received either no service or a lesser service, were fearful of denial of treatment in the future if they complained, and had no access to preferential treatment. The differences between the disadvantaged and advantaged in Australia's health system were less marked as the delivery of public health care for poorer Australians is not dependent on fund raising or charity, nor does it generally rely on co- payments (outside of some tests, some pharmaceuticals, 
disposable goods and aids) and therefore does not present the same conflicts as the Korean system (Jeong, 2011).

Respect for life and philanthropy, encapsulated in the Korean code of ethics, were personal moral positions and professional values that were very important to Korean social workers. These values were most challenged when they felt sympathy for their patients - for example "the destitute and alone” - who did not have the financial resources to pursue treatment, were ineligible or, for example, were compelled to donate a body to the hospital due to an inability meet funeral costs.

Macro structures outside of health, particularly those immigration policies on refugees, asylum seekers or illegal immigrants were key sources of distress for hospital social workers in both countries. Because eligibility for services differed from those available to citizens, the relative poverty experienced by these people meant social workers in South Korea and Australia were unable to respond adequately to their needs, if at all. Inequitable treatment was a highly problematic feature of their work as some people were both ineligible, unable to pay for private care or faced a precarious future. As one Australian social worker recounted:

“...part of the immigration is about having AIDS testing and so there's an automatic referral if a patient is diagnosed with HIV/ AIDS which could mean that the visa is then withdrawn, and the status to remain in the country is gone and then they are left to return to their home country, regardless of whether they can get that treatment there... But even just while he remained in the country in order to access the medication and to be able get services you know, the bill for hospital stay. So I had two patients who were both diagnosed with AIDS but taking completely different routes even though they were of similar 
ages, and so as far as the support to the individual patient, this particular one also had a young child and a wife.”

This distressing example was one that haunted the social worker. Moral distress brought about by the inability to meet the needs of those people without citizenship status was also reflected in the Korean social workers’ experiences.

"For me, since I work in a public institution, various types of patients, especially illegal immigrants come. As soon as they come, the financial problem arises... it is difficult to provide sufficient medical care to these illegal immigrants. Since we are very limited in providing medical care to those people, we have no option but to advise them to go back to their countries. But since, in most cases, their countries are not in a good financial situation and they mostly came to earn money, advising them to go back is no different from a death sentence.”

It was these situations that elicited the most distress for participating social workers because the inability to assist the disenfranchised, social injustice and a failure to provide adequate healthcare contrasted so sharply with their professional and personal positions of morality. Moral distress felt about these cases was not simply a result of the allocation of resources rather it was strongly connected to values, breaches of human rights and discriminatory policies.

\section{Coping and codes}

Participants in both countries described a number of coping strategies to manage the distress caused by moral conflicts between what they could and could not deliver, their professional values, institutional climates and macro policies. These were consultation, supervision, peer support, deferring to organisational procedures, resistance (even when threatened), simply doing what they could, rationalisation and 
actively using their social work codes of ethics. The participants used social work codes in two distinct ways, to mediate dilemmas and to validate a collective experience of shared values reinforcing their social work identity in those scenarios where moral distress was experienced.

In all cases, social workers reported consultation with colleagues or professional supervision as the greatest sources of support when dealing with complex ethical challenges and moral conflicts that induced moral distress. This finding mirrored that reported in an earlier Australian study on sources of support in situations of ethical conflict (McAuliffe \& Sudbury, 2005). Korean social workers regularly referred to their code whereas Australian social workers tended to do so only when their values were challenged. Korean social workers were often required to defend their decisions before committees which may emphasize the importance and relevance of their code of ethics as a moral and practical document that gave legitimacy to particular actions. Although Australian social workers found they did need to defend their decisions in multidisciplinary team meetings, they rarely referred directly to their code in these situations. Most participant social workers reported using their codes during professional supervision for ethical decision making which involved managing risk, dealing with conflict over courses of action, determining capacity and consent, when they were unsure what to do, when boundary issues arose, and when they faced a new dilemma. Importantly, they also referred to their code when their values clashed with that of the organisation and moral conflict and distress was experienced. Australian and Korean social workers identified hospital codes, rules and protocols as more influential than codes of ethics as working documents in daily practice (until there was a clash of values and moral concerns). 
A strong sense of professionalism and their social work codes of ethics helped participants in both groups stay committed to their positions when challenged, and referred to their education as preparatory for a strong social work identity and value commitment. An inability of the social workers to act the way they felt they should were sometimes rationalised to make sense of no-win situations. As previously suggested, rational processes do not necessarily ease moral concerns (Bauman, 1998; Gray 2010). For example, one Korean social worker said:

"I understand $100 \%$ that is it discriminating against people who cannot afford the service and that one takes the people who can afford it. 'Is it unethical?' It would be ideal if the government takes the responsibility and gives people equal opportunity. For example, I'm not sure if this is a good example, say, there's a person who needs a car. But if the person cannot afford a car and there is an Excel, the old Excel model, and if a wealthy person has a Sonata [Excel as an example of outdated and cheaper car compared to Sonata], is this unethical? This thought was lingering for a while in my mind. I thought 'if the person really needs a car whether it's an Excel or a Sonata, wouldn't it be ideal to at least be granted a car and get at least the minimum support although it's a junk car?" So again there is a contradictory thought." (Korean participant)

Distress was experienced in relation to unresolvable moral problems and, in this sample, participants in both countries used words such as 'uncomfortable', 'distressing', 'shock', and 'emotive’ when describing their responses. Moral distress was also noted in tone and body language when participants described some situations such as those where social workers were morally compromised by others, where treatment would not be provided and where people were in dire circumstances due to poverty or citizenship status and future harm would likely eventuate. 


\section{Discussion}

Many of the findings of this study are similar to those previously identified in social work practice in the welfare and child protection sectors where moral distress is associated with value clashes and structural factors that limit what social workers can do (Abramovtiz, 2005; Austin et al., 2005; McAuliffe, 2005; Weinberg, 2009, 2010, 2016). The participants in this study experienced distress from moral conflicts caused by unjust social policies, institutional cultures and interdisciplinary value conflicts. These range from discharging patients to unsafe or harmful environments through to coercive pressure to make unjust decisions. The differences in experience highlighted the intensity of the challenges faced by Korean social workers in this study compared to that of the Australian social workers.

Three key issues came out of this exploration. The first is the relationship between moral distress felt by social workers in organisational cultures where corruption and conflicts of interest are commonplace. The experience of Korean social workers transcended other experiences found in the literature as they had to negotiate corrupt practices and to bear the consequences of maintaining ethical and moral positions which included direct threats for one social worker. Lützén and Kvist (2012) claim a hypothetical link between moral sensitivity, moral distress and ethical climate. Ethical climates where a unifying ethical culture does not exist or institutional codes are ignored and where conflicts of interest and dual relationships are commonplace may present more than a hypothetical relationship with moral distress.

The second, relevant to practice in both countries, suggests that social policies cannot be understood in silos. How social workers are able to fulfil their moral obligations at the coalface is not simply a product of health policy or institutional practice rather the interconnectedness of policies across sectors such as health, 
welfare, immigration and so on that together impinge on social justice and respect for persons. This interconnectedness and their impact is a significant factor in the moral distress experienced by these participants. An inability to provide equitable services to refugees, asylum seekers and illegal migrants caused the greatest distress.

Immigration policies ultimately determined which treatments were funded and who could receive them. In contemporary neoliberal and privatised environments the allocation of funding or the availability of private resources means that some citizens are excluded from certain treatments. As Weinberg (2009) suggests, moral distress functions as a catalyst for social workers to make sense of the personal and the political. Without distress, moral problems might go unnoticed, posing risks of mechanistic work and subsumption by organisational expectations even where values conflict.

Thirdly, social workers in this study referred to their professional codes to resolve ethical dilemmas and to assist in decision making processes. Of significance was the use of codes of ethics as a means of reconnecting to a collective social work identity and shared values. Rather than merely becoming victims to helplessness and despair as reported in some studies (Pauly, Varcoe and Storch, 2012), codes seemed to have a practical function in reinforcing their moral position and played a role in easing distress. Connecting to the explicitly stated values in social work codes that are critiqued by Gray (2010) and others (Bauman, 1993) for their codification shifts personal responsibility towards a shared and therefore justified position that does appear to have some role in addressing the moral distress experienced by some social workers. Although referring to codes did not entirely eliminate the distress felt about particular situations, the use of codes supported by professional supervision and collegial support shifted the impact from the individual to the collective and provided 
moral justification for individual reactions that reassured rather than engendered a sense of failure. This finding reinforces suggestions that social work codes may need to emphasise values as strongly as other components of codes of ethics. Weinberg and Teram (2016) suggest codes should shift away from a technocratic and procedural focus and accentuate inspirational values. Perhaps rather than sacrificing one function for another, a dual focus on providing guidance with ethical decision making and emphasising values is important for practice.

As an exploratory study, the findings identified here highlight some key issues that require further research attention. Methodologies that allow in-depth exploration of moral distress in hospital social work can give more insight into the personal and institutional consequences, how the chronicity of distress affects social workers' wellbeing, how codes of ethics are used to alleviate moral distress, and whether codes of ethics supported by professional supervision and collegial support can sustain practitioners in the long term. This is particularly important given the changing contexts of health delivery in many countries, the impact of globalization on healthcare and social work practice, and the revision of existing social work codes and the development of new ones.

The conspicuous differences between the Australian and Korean experiences was attributable to the systems in which the social workers worked. The surprising element for both groups was just how influential social policies were and how direct that influence was on front-line hospital social work and on the experience of moral distress. How a country organises its health system and other related policy areas can create climates in which moral distress can be amplified.

There is much discussion in the literature about individual agency and an inability to act. Weinberg (2009, p. 146) tempered this view by pointing out that "individuals 
are neither completely autonomous nor are they purely victims of systems”. There was evidence that social workers in this study did act, did not always perceive themselves as helpless and were realistic about the change they could effect. However, they still suffered the emotional impact of unresolvable situations. They acted by doing the best they could, taking a stance, resisting or holding out as long as they could, exerting their professional expertise, and using their codes of ethics to defend their decisions and a tool to reconnect to collective social work values.

\section{Limitations}

This study was exploratory and the sample size was small. As such the findings are not generalizable which is not a major goal in qualitative inquiry. While a contained focus group design for an exploratory study is appropriate (Wilkinson, 1998), collecting data from other sources such as in-depth interviews may have strengthened the findings. Further interviews with participants and additional focus groups at other sites would extend this research and allow further investigation of many of the issues identified in this study.

The limitations of a group setting meant social workers may either have felt constrained by the group setting or feelings of distress may have been mitigated by the collegial support within the group. Written responses provided by participants at the conclusion of the focus groups provided opportunities for private disclosure and in both groups, participants reported that they were appreciative of the opportunity to express their views.

\section{Conclusion}

The study draws particular attention to several issues. In particular, the findings highlighted the very real impact of ethical cultures in the workplace and the interconnectedness of social policies and their influence on moral distress at the 
hospital practice level. The moral distress experienced was not simply a response to the distribution of resources rather to those macro policies and institutional practices that exacerbate social injustice, discrimination or unethical practices. More understanding is needed about the experiences of all health social workers, particularly in those countries where health systems contribute more readily to unresolvable situations that lead to moral distress, how the profession and health institutions can support them, and how educators prepare students for practice in such environments. Values expressed in social work codes of ethics do appear to play some role in shifting the consequences of moral distress from the individual to a shared value base. The findings suggest that it is important for hospital social work departments to increase their awareness of potential moral distress amongst their social workers and to develop strategies relevant to their context that are inclusive of reinforcing shared values to address any moral distress experienced. Social work research needs to expand this knowledge, and explore how to promote hopeful practice rather than helplessness in challenging practice contexts such as hospitals.

\section{References}

AASW, (2010). Code of Ethics, Australia, Australian Association of Social Workers. Accessed November 30, 2014 at http://www.aasw.asn.au/document/item/1201. Abramovitz, M. (2005). The largely untold story of welfare reform and the human services. Social Work, 50, (2) 175-186.

Austin, W., Rankel, M., Kagan, L., Bergum, V., \& Lemermeyer, G. (2005). To stay or to go, to speak or stay silent, to act or not to act: Moral distress as experienced by psychologists. Ethics \& Behavior, 15(3), 197-212. doi: 10.1207/s15327019eb1503_1 
Banks, S. (1998). Professional Ethics in Social Work- What Future? British Journal of Social Work, 28(2), 213-231.

Banks, S. (2003). From oaths to rulebooks: a critical examination of codes of ethics for the social professions. European Journal of Social Work, 6(2), 133-144. doi: $10.1080 / 1369145032000144403$

Banks, S. (2012). Ethics and values in social work. London: Macmillan Press. Bauman, Z. (1998). What prospects of morality in times of uncertainty? Theory, Culture and Society, 15(1), 11-22.

Bauman, Z. (1993). Postmodern ethics. Oxford: Blackwell.

Bisman, C. (2004). Social work values: The moral core of the profession. British Journal of Social Work, 34, 109-123. doi: 10.1093/bjsw/bch008

Braun, V., \& Clarke, V. (2006). Using thematic analysis in psychology. Qualitative Research in Psychology, 3, 77-101.

Brazil, K., Kassalainen, S., Ploeg, J., \& Marshall, D. (2010). Moral distress experienced by health care professionals who provide home-based palliative care. Social Science \& Medicine, 71(9), 1687-1691. doi: http://dx.doi.org/10.1016/j.socscimed.2010.07.032 Brown, L. D. (2003). Comparing health systems in four countries: Lessons for the United States. American Journal of Public Health, 93(1), 52-56.

Cacace, M., Ettelt, S., Mays, N., \& Nolte, E. (2013). Assessing quality in crosscountry comparisons of health systems and policies: Towards a set of generic quality criteria. Health policy (Amsterdam), 112(1-2), 156-162.

Carinci, F., Van Gool, K., Mainz, J., \& Veillard, J. (2015). Towards actionable international comparisons of health system performance: expert revision of the OECD 
framework and quality indicators. International journal for quality in health care, 27(2), 137-146.

Congress, E., \& McAuliffe, D. (2006). Social work ethics: Professional codes in Australia and the United States. International Social Work, 49(2), 151-164. doi: $10.1177 / 0020872806061211$

Denzin, N. (1994). The art and politics of interpretation. In N. Denzin \& Y. Lincoln (eds.) Handbook of qualitative research (p. 500-15), London: Sage.

Duckett, S. J. and Willcox, S. (2011). The Australian Health-Care System, (4th ed.). Melbourne, Oxford University Press.

Fine, M., \& Teram, E. (2009). Believers and skeptics: Where social workers situate themselves regarding the Code of Ethics. Ethics \& Behavior, 19(1), 60 - 78. doi: $10.1080 / 10508420802623682$

Fine, M., \& Teram, E. (2013). Overt and covert ways of responding to moral injustices in social work practice: Heroes and mild-mannered social work bipeds. British Journal of Social Work, 43(7), 1312-1329. doi: 10.1093/bjsw/bcs056 Fronek, P., \& Kendall, M. B. (2016). The impact of Professional Boundaries for Health Professionals (PBHP) training on knowledge, comfort, experience, and ethical decision-making: a longitudinal randomized controlled trial. Disability and Rehabilitation, 1-8. doi:10.1080/09638288.2016.1236152

Gallina, N. (2010). Conflict between professional ethics and practice demands: Social work perceptions. Journal of Social Work Values and Ethics, 7(2), 1-9.

Gray, M. (2010). Postmodern ethics. In Ethics and value perspectives in social work. London: Palgrave. 
Greenslade, L., McAuliffe, D., \& Chenoweth, L. (2015). Social workers' experiences of covert workplace activism. Australian Social Work, 68(4), 422-437. doi:10.1080/0312407X.2014.940360

Habermas, J. (1990). Moral consciousness and communicative action. Cambridge: Polity Press.

Jameton, A. (1984). Nursing practice: The ethical issues. Englewood Cliffs, NJ: Prentice Hall.

Jeong, H. S. (2011). Korea's National Health Insurance - Lessons from the past three decades, Health Affairs, 30(1), 136-144.

Kälvemark, S., Höglund, A., Hansson, M., Westerholm, P., \& Arnetz, B. (2004). Living with conflicts - ethical dilemmas and moral distress in the health care system. Social Science and Medicine, 58(6), 1075-1084.

KASW, (2001) Code of Ethics, South Korea, Korean Association of Social Workers. Accessed December 4, 2014 at http://www.welfare.net/site/ViewMoralCode.action. Kim, D. S. (2010). Introduction: Health of the health care system in Korea, Social Work in Public Health, 25(2), 127-141.

Lützén, K., \& Kvist, B. E. (2012). Moral distress: A comparative analysis of theoretical understandings and inter-related concepts. HEC Forum, 24(1), 13-25. doi: 10.1007/s10730-012-9178-9 Lynch, D., \& Forde, C. (2016). ‘Moral distress’ and the beginning practitioner: preparing social work students for ethical and moral challenges in contemporary contexts. Ethics and Social Welfare, 10(2), 94-107. doi:

$10.1080 / 17496535.2016 .1155634$ 
Mänttäri-van der Kuip, M. (2016). Moral distress among social workers: The role of insufficient resources. International Journal of Social Welfare, 25(1), 86-97. doi: 10.1111/ijsw.12163

McAuliffe, D. (2005). I'm still standing: Impacts and consequences of ethical dilemmas for social workers in direct practice, Journal of Social Work Values and Ethics, 2(1), 1-11.

McAuliffe, D. \& Sudbury, J. (2005). Who do I tell? Support and consultation in cases of ethical conflict, Journal of Social Work, 5(1), 25-33.

McAuliffe, D. (2014). Interprofessional ethics: Collaboration in the social, health and human services, London: Cambridge University Press.

Meeuwisse, A., Scaramuzzino,R., \& Swärd, H. (2011). Everyday realities and visionary ideals among social workers in the Nordic countries: A Matter of specialization and work tasks? Nordic Social Work Research, 1(1): 5-23.

Mukherjee, D., Brashler, R., Savage, T. A., \& Kirschner, K. L. (2009). Moral distress in rehabilitation professionals: results from a hospital ethics survey. $P M \& R$ : The Journal Of Injury, Function, And Rehabilitation, 1(5), 450-458.

Nathaniel, A. (2002, Spring). Moral distress among nurses. The American Nurses Association Ethics and Human Rights Issues Updates, 1(3). Retrieved September 8, 2004, from http://www.nursingworld.org/ethics/update/vol1no3a.htm\#moral O’Reilly, M., \& Parker, N. (2012). ‘Unsatisfactory Saturation’: A critical exploration of the notion of saturated sample sizes in qualitative research. Qualitative Research, 13(2), 190-197.

Pauly, B. M., Varcoe, C., \& Storch, J. (2012). Framing the issues: Moral distress in health care. [journal article]. HEC Forum, 24(1), 1-11. doi: 10.1007/s10730-0129176-y 
Pockett, R., \& Beddoe, L. (2015). Social work in health care: An international perspective. International Social Work, 60(1), 126-139.

doi:10.1177/0020872814562479

Reamer, F. G. (2005). Ethical and legal standards in social work: Consistency and conflict. Families in Society, 86(2), 163-169.

Seah, D. S. E., Cheong, T. Z., \& Anstey, M. H. R. (2013). The hidden cost of private health insurance in Australia, Australian Health Review, 37(1), 1-3.

Sunderland, N., Catalano, T., Kendall, E., McAuliffe, D. \& Chenoweth, L. (2010). Exploring the concept of moral distress with community-based researchers: An Australian study, Journal of Social Service Research, 37(1), 73-85.

Weinberg, M. (2009). Moral distress. Canadian Social Work Review, 26(2), 139-151.

Weinberg, M. (2010). The social construction of social work ethics: Politicizing and broadening the lens. Journal of Progressive Human Services, 21(1), 32-44. doi:

$10.1080 / 10428231003781774$

Weinberg, M., \& Campbell, C. (2014). From codes to contextual collaborations:

Shifting the thinking about ethics in social work. Journal of Progressive Human

Services, 25(1), 37-49. doi: 10.1080/10428232.2014.856739

Wilkinson, S. (1998). Focus group methodology: a review. International Journal of Social Research Methodology, 1(3), 181-203. 